\title{
Retreatment with Bendamustine-Bortezomib-Dexamethasone in a Patient with Relapsed/Refractory Multiple Myeloma
}

\author{
Claudio Cerchione, Davide Nappi, Maria Di Perna, Irene Zacheo, Anna Emanuele Pareto, \\ Marco Picardi, Lucio Catalano, and Fabrizio Pane
}

Hematology, University Federico II, Via Pansini 5, 80131 Napoli, Italy

Correspondence should be addressed to Claudio Cerchione; claudiocerc@hotmail.com

Received 18 July 2016; Accepted 12 October 2016

Academic Editor: Tatsuharu Ohno

\begin{abstract}
Copyright ( $\odot 2016$ Claudio Cerchione et al. This is an open access article distributed under the Creative Commons Attribution License, which permits unrestricted use, distribution, and reproduction in any medium, provided the original work is properly cited.

The clinical management of relapsed/refractory multiple myeloma and the correct choice of the most suitable therapy in heavily pretreated and fragile patients are tough clinical issues for clinicians. In advanced phases of disease, the choice of available therapies becomes very poor, and the retreatment with previously adopted and effective therapy, although unpredictable, could be an effective option. In this report, we describe the clinical history of a patient, previously treated with 9 lines of therapy, refractory to bortezomib and IMIDs, for whom the retreatment with bendamustine resulted in a stable disease with good quality of life.
\end{abstract}

\section{Introduction}

In advanced multiple myeloma, the choice of the treatment can be difficult, as therapeutic options decrease over time. Both new combinations of previously used drugs and retreatment with a previously adopted and effective therapy can be taken into consideration in patients showing persistent chemosensitivity. In this report, we describe the case of a heavily pretreated patient, refractory to bortezomib and IMIDs, with clinical benefit after retreatment with bendamustine.

\section{Case Presentation}

In June 2009, this male patient was 67 years old and was diagnosed with IgG $\lambda$ stage IIIA multiple myeloma (MM). FISH analysis was performed at diagnosis, and it showed negativity for the most frequent alterations ( $t(11$; 14), $t(4 ; 14)$, del13q, and del17p). First-line therapy was 7 cycles of thalidomide-dexamethasone (TD), followed by radiotherapy on T2. In March 2010 progressive bone disease was detected by MRI of the spine showing multiple cervical and dorsal osteolytic lesions. Thus, second line of bortezomib-desametasone (VD), together with zoledronic acid, was performed for 5 cycles, obtaining a partial response.
A first ASCT, preceded by thiotepa/melphalan conditioning regimen, was performed in December 2010 leading to a partial response. After a period with stable clinical conditions, in April 2011, disease progression was documented by the increase of the serum monoclonal component (sMC): the patient was treated with 4 courses of lenalidomidedexamethasone (RD), but the disease progressed. Therefore, a combination of melphalan-lenalidomide-dexamethasone (MRD) was performed for 3 cycles in September 2011, again followed by disease progression, determined by sMC increase. At the same time, PET/CT performed for neck pain revealed multiple osteolytic lesions: the most dangerous (C2) was treated with tomotherapy ( $40 \mathrm{~Gy}$ total). Thus, 2 cycles of cyclophosphamide-doxorubicin-dexamethasone (CED) regimen were attempted (1), but the disease was still refractory. Hence, a bendamustine-bortezomib-dexamethasone (BVD) regimen was administered (bendamustine $90 \mathrm{mg} / \mathrm{sqm}$ at days 1 and 2, bortezomib $1.3 \mathrm{mg} / \mathrm{sqm}$ at days $1,4,8$, and 11 , dexamethasone $20 \mathrm{mg}$ at days $1,2,4,5,8,9,11$, and 12 , and pegfilgrastim $6 \mathrm{mg}$ at day +4$)(2,3$, and 4) for 6 cycles, resulting in a partial response, followed by a second ASCT, preceded by thiotepa/melphalan conditioning regimen. In February 2014, a further sMC increase suggested disease progression, and the patient was treated with bortezomiblenalidomide-dexamethasone (VRD) for 6 cycles with the 
TABle 1: Patient's history.

\begin{tabular}{lccc}
\hline Line & Regimen & Cycle $\left(\mathrm{n}^{\circ}\right)$ & Responses \\
\hline 1 & Thalidomide-dexamethasone + RT & 7 & Progressive disease \\
2 & Bortezomib-dexamethasone & 5 & Partial response \\
3 & First auto-BMT (thiotepa-melphalan) & $/$ & Stable disease \\
4 & Lenalidomide-dexamethasone & 4 & Progressive disease \\
5 & Melphalan-lenalidomide-dexamethasone & 3 & Progressive disease \\
6 & Doxorubicin-cyclophosphamide-dexamethasone & 2 & Progressive disease \\
7 & Bendamustine-bortezomib-dexamethasone & 6 & Partial response \\
8 & Second auto-BMT (thiotepa-melphalan) & $/$ & Stable disease \\
9 & Bortezomib-lenalidomide-dexamethasone & 6 & Progressive disease \\
10 & Bendamustine-bortezomib-dexamethasone & 7 & Stable disease \\
11 & Pomalidomide-dexamethasone & 4 & Progressive disease \\
\hline
\end{tabular}

result of progressive disease. In November 2014, for disease progression confirmed also by PET/CT scan (Table 1), even considering cardiovascular comorbidities, BVD-retreatment was chosen as tenth line. The patient switched to a stable disease status and clinical conditions were relatively fit for more than one year. The treatment was well tolerated: the only toxicities were grade 2 anemia and grade 3 thrombocytopenia, while severe neutropenia was effectively prevented with pegfilgrastim prophylaxis ( $6 \mathrm{mg}$ at day +4 of every courses). No extrahematological side effects were revealed.

Due to further sMC increase, in December 2015, 4 courses of pomalidomide-dexamethasone were attempted, in a palliative intent, but the patient died in July 2016.

\section{Discussion}

After the advent of proteasome inhibitors, international guidelines agree on first-line treatment strategy for ASCTeligible and noneligible patients [1-3]. However, selecting and managing the correct therapy for a patient with rrMM it is still a tough task for the hematologist, as, after many relapses, available therapeutic options are scanty. A commonly adopted strategy consists in retreating the patient with the same molecules used previously, choosing those which showed the best response or considering new drug combinations, even if in previous administrations single drugs showed to be ineffective [4-9].

This strategy seems particularly successful in patients who show persistent chemosensitivity, as in our case, who obtained an overall survival longer than 7 years, which can be considered as an impressive result in a 67-year-old patient affected by MM.

Bendamustine is a well-tolerated agent with a double mechanism of action, alkylating and antimetabolite, with proved effectiveness in treatment of relapsed/refractory [10, 11] and newly diagnosed multiple myeloma [12, 13] and in a relapsing/refractory setting [14-19]. In rrMM it can be used as single agent combined to dexamethasone, but a synergistic effect has been demonstrated when associated with bortezomib.

Bendamustine showed significant efficacy also in a selected setting of patients, such as those who became refractory to bortezomib and IMIDs or multirelapsed after single or double ASCT, demonstrating also an effective opportunity as a bridge to ASCT [10]. To the best of our knowledge, BVD-retreatment for relapsing/refractory MM is still not consolidated, but, as in our case, it could be considered an effective choice in heavily pretreated patients without significant therapeutic options, in a context of a welltolerated palliative treatment with good quality of life.

\section{Competing Interests}

The authors declared that there are no competing interests.

\section{References}

[1] M. Engelhardt, E. Terpos, M. Kleber et al., "European myeloma network recommendations on the evaluation and treatment of newly diagnosed patients with multiple myeloma," Haematologica, vol. 99, no. 2, pp. 232-242, 2014.

[2] K. C. Anderson, M. Alsina, D. Atanackovic et al., "NCCN guidelines insights: multiple myeloma, version 3. 2016," Journal of the National Comprehensive Cancer Network, vol. 14, no. 4, pp. 389-400, 2016.

[3] G. Barosi, G. Merlini, A. Billio et al., "SIE, SIES, GITMO evidence-based guidelines on novel agents (thalidomide, bortezomib, and lenalidomide) in the treatment of multiple myeloma," Annals of Hematology, vol. 91, no. 6, pp. 875-888, 2012.

[4] A. K. Stewart and A. Palumbo, "Treatment of relapsed multiple myeloma," The New England Journal of Medicine, vol. 372, no. 18, pp. 1774-1775, 2015.

[5] A. K. Nooka, E. Kastritis, M. A. Dimopoulos, and S. Lonial, "Treatment options for relapsed and refractory multiple myeloma," Blood, vol. 125, no. 20, pp. 3085-3099, 2015.

[6] J. Bladé, L. Rosiñol, and C. Fernández de Larrea, "How I treat relapsed myeloma," Blood, vol. 125, no. 10, pp. 1532-1540, 2015.

[7] P. G. Richardson, W. Xie, S. Jagannath et al., "A phase 2 trial of lenalidomide, bortezomib, and dexamethasone in patients with relapsed and relapsed/refractory myeloma," Blood, vol. 123, no. 10, pp. 1461-1469, 2014.

[8] C. Cerchione, M. Lucignano, F. Pane, and L. Catalano, "Salvage therapy with pegylated liposomial doxorubicin-based regimen in relapsed/refractory multiple myeloma: comments to the article by Romano et al," European Journal of Haematology, vol. 96, no. 5, p. 544, 2016. 
[9] C. Cerchione, L. Catalano, A. E. Pareto, M. Picardi, and F. Pane, "Pegfilgrastim in primary prophylaxis of febrile neutropenia during chemotherapy of relapsed and refractory multiple myeloma: a real-life experience," Supportive Care in Cancer, vol. 23, no. 2, pp. 301-302, 2014.

[10] C. Cerchione, L. Catalano, A. E. Pareto et al., "Bendamustine in the management of relapsed and refractory multiple myeloma: a regional real-life experience," Hematologica, vol. 100, supplement 1, 2015.

[11] S. Lentzsch, "Bendamustine: the remedy that came in from the cold," Blood, vol. 123, no. 7, pp. 948-950, 2014.

[12] M. Gentile, E. Vigna, A. G. Recchia et al., "Bendamustine in multiple myeloma," European Journal of Haematology, vol. 95, no. 5, pp. 377-388, 2015.

[13] W. Pönisch, M. Andrea, I. Wagner et al., "Successful treatment of patients with newly diagnosed/untreated multiple myeloma and advanced renal failure using bortezomib in combination with bendamustine and prednisone," Journal of Cancer Research and Clinical Oncology, vol. 138, no. 8, pp. 1405-1412, 2012.

[14] M.-V. Mateos, A. Orio, L. Rosiñol et al., "Bendamustine, bortezomib and prednisone for the treatment of patients with newly diagnosed multiple myeloma: results of a prospective phase 2 Spanish/PETHEMA trial," Haematologica, vol. 100, no. 8, pp. 1096-1102, 2015

[15] R. Fenk, M. Michael, F. Zohren et al., "Escalation therapy with bortezomib, dexamethasone and bendamustine for patients with relapsed or refractory multiple myeloma," Leukemia and Lymphoma, vol. 48, no. 12, pp. 2345-2351, 2007.

[16] G. Damaj, F. Malard, C. Hulin et al., "Efficacy of bendamustine in relapsed/refractory myeloma patients: results from the French compassionate use program," Leukemia and Lymphoma, vol. 53, no. 4, pp. 632-634, 2012.

[17] M. Offidani, L. Corvatta, L. Maracci et al., "Efficacy and tolerability of bendamustine, bortezomib and dexamethasone in patients with relapsed-refractory multiple myeloma: a phase II study," Blood Cancer Journal, vol. 3, no. 11, article e162, 2013.

[18] H. Ludwig, H. Kasparu, C. Leitgeb et al., "Bendamustinebortezomib-dexamethasone is an active and well-tolerated regimen in patients with relapsed or refractory multiple myeloma," Blood, vol. 123, no. 7, pp. 985-991, 2014.

[19] P. Rodon, C. Hulin, B. Pegourie et al., "Phase II study of bendamustine, bortezomib and dexamethasone as second-line treatment for elderly patients with multiple myeloma: the Intergroupe Francophone du Myelome 2009-01 trial," Haematologica, vol. 100, no. 2, pp. e56-e59, 2015. 


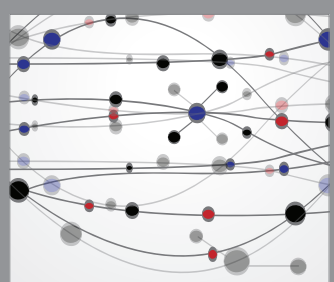

The Scientific World Journal
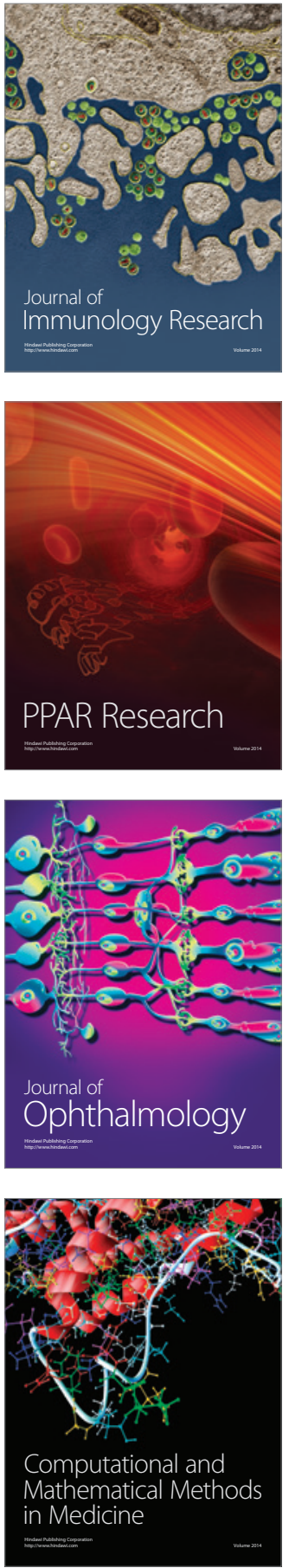

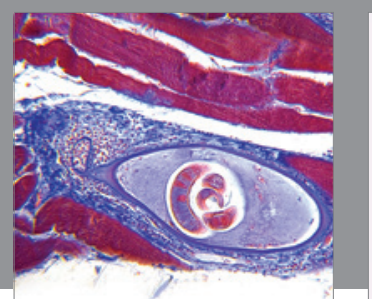

Gastroenterology Research and Practice

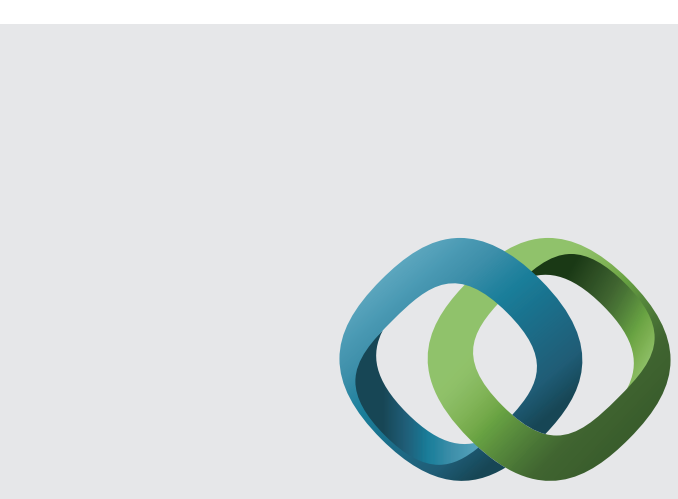

\section{Hindawi}

Submit your manuscripts at

http://www.hindawi.com
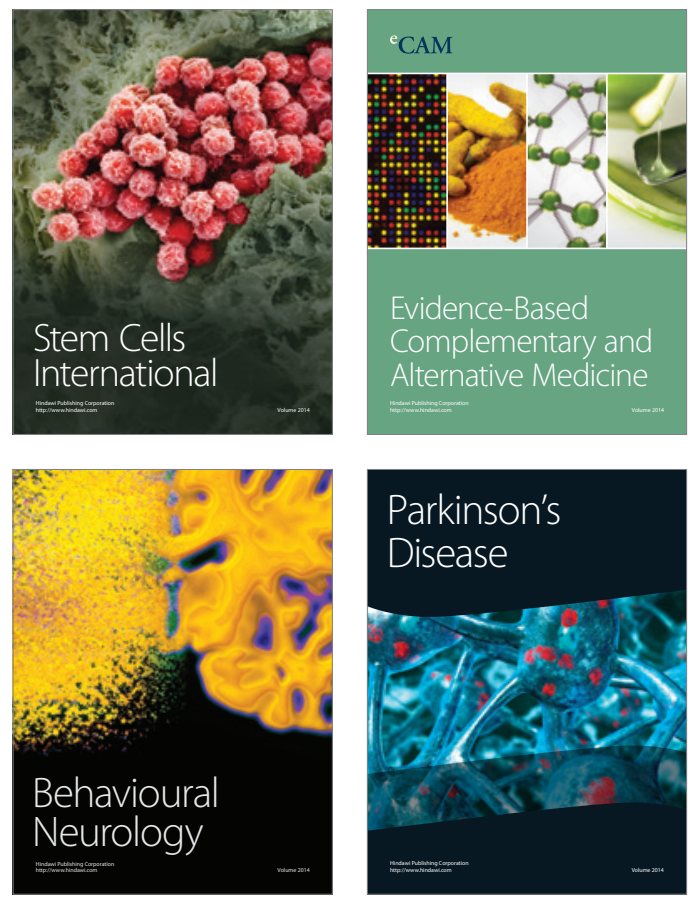
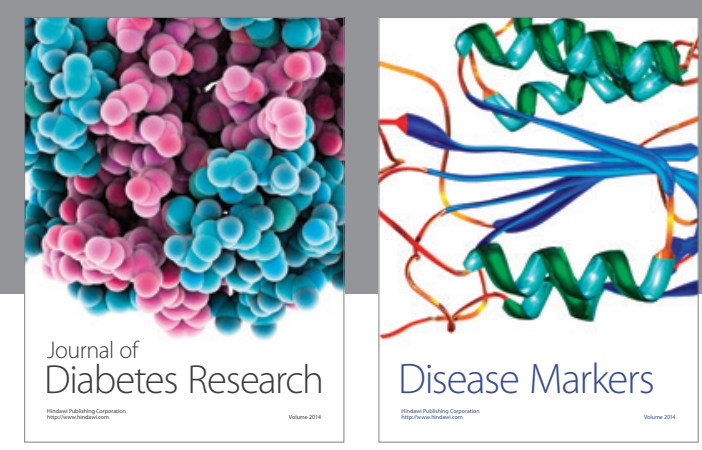

Disease Markers
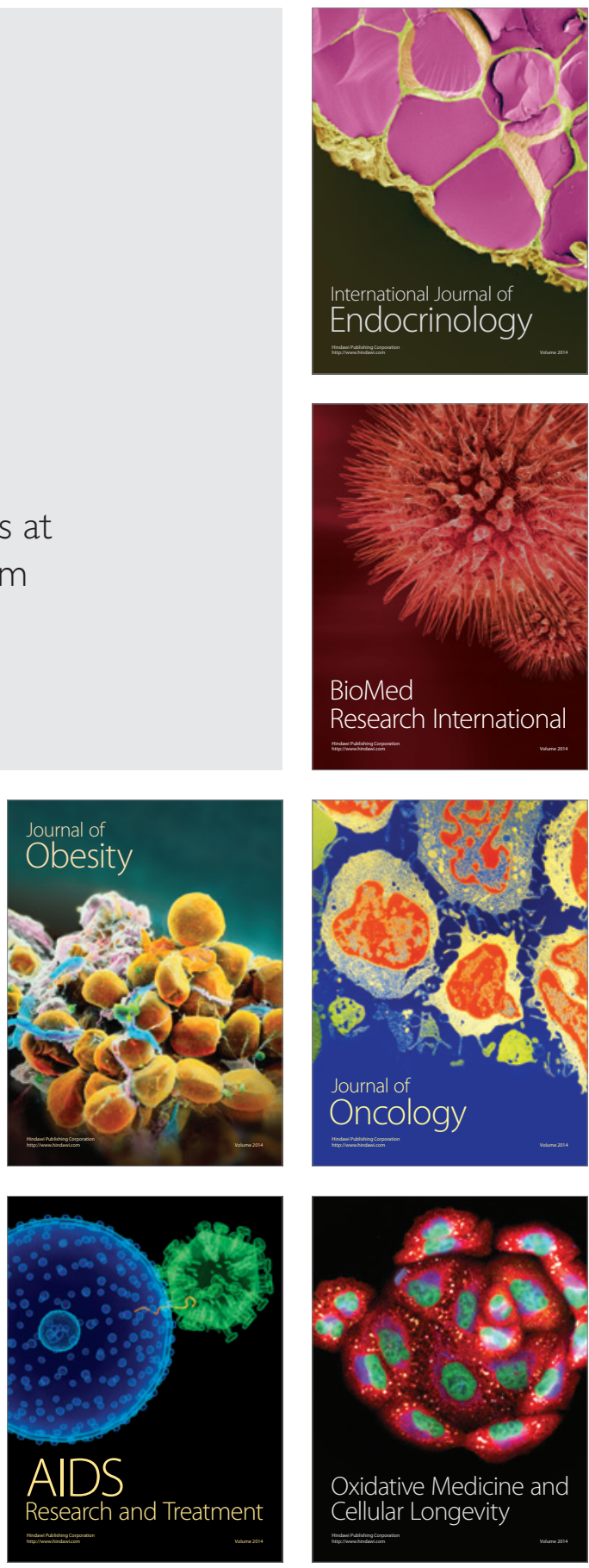